SHORT REPORT

\title{
Matrilin-3 mutations that cause chondrodysplasias interfere with protein trafficking while a mutation associated with hand osteoarthritis does not
}

\author{
C Otten, R Wagener, M Paulsson, F Zaucke
}

J Med Genet 2005;42:774-779. doi: 10.1136/jimg.2004.029462

\begin{abstract}
Several mutations in the extracellular matrix protein matrilin3 cause a heterogeneous disease spectrum affecting skeletal tissues. We introduced three disease causing point mutations leading to single amino acid exchanges (R116W, T298M, C299S) in matrilin-3 and expressed the corresponding proteins in primary articular chondrocytes to elucidate pathogenic mechanisms at the cellular level. Expression levels, processing, and the secretion pattern of a mutation linked to hand osteoarthritis (T298M) were similar to the wildtype protein, whereas the two other mutants were poorly expressed and hardly detectable in supernatants of transiently transfected cells. Using immunofluorescence staining, we demonstrated that mutants R116W and C299S are retained and accumulate within the endoplasmatic reticulum (ER). Their further trafficking to the Golgi compartment seems to be disturbed, whereas T298M is secreted normally. In cells transfected with the wildtype and T298M constructs, a matrilin-3 containing filamentous network was formed surrounding the cells, whereas in the case of R116W and C299S such structures were completely absent. These observations are similar to those for mutations in the cartilage oligomeric matrix protein (COMP) leading to multiple epiphyseal dysplasia and pseudoachondroplasia suggesting that retention and accumulation of cartilage proteins in the ER might be a general mechanism involved in the pathogenesis of chondrodysplasias.
\end{abstract}

$\mathrm{T}$ he matrilin family of extracellular matrix (ECM) proteins consists of four members and is characterised by a modular domain structure made up of von Willebrand factor A (vWA) domain(s), epidermal growth factor (EGF)like domain(s), and a C-terminal $\alpha$-helical coiled coil domain promoting oligomerisation. The four members are differentially expressed with matrilin- 1 and -3 being predominantly found in skeletal tissues, while matrilin-2 and -4 are more widely distributed. ${ }^{1}$ In contrast to the other family members with two vWA domains, monomeric matrilin-3 consists only of a single vWA domain followed by four EGF-like domains and the coiled coil domain. ${ }^{2}$ Via this coiled coil domain, matrilin-3 is able to form homotetramers as well as heterotrimers and -tetramers with matrilin- $1 .{ }^{3-5}$ In addition to hetero-oligomeric assembly with certain other family members, all matrilins have been shown to interact with a variety of both collagenous and non-collagenous proteins, suggesting a function as adaptor proteins within the ECM. ${ }^{6-10}$ Moreover, the formation of filamentous structures in the pericellular matrix of cultured cells has been described for all four matrilins. ${ }^{41-13}$

The targeted disruption of each of the genes coding for matrilin-1, -2, and -3 did not result in an obvious phenotype, leading to the hypothesis that the loss of one matrilin can be compensated for by other family members, although those are not upregulated at the RNA level. ${ }^{14-17}$

So far, patient mutations have been described only in matrilin-3 and linked to clinically defined skeletal defects. Several mutations in the $\beta$ strands of the single vWA domain of matrilin-3 are associated with multiple epiphyseal dysplasia (EDM5; MIM 607078) 18 $^{19}$ and bilateral hereditary microepiphyseal dysplasia (BHMED) which is distinct from common MED. ${ }^{20}$ MED is characterised by delayed and irregular ossification of the epiphysis and early onset osteoarthritis, while BHMED patients have small epiphyses in the hip and knee joint. Not only mutations in matrilin-3 but at least five other genes potentially causing MED have been identified, including those coding for all three chains of collagen type IX, ${ }^{21-23}$ the cartilage oligomeric matrix protein (COMP) ${ }^{24}$ and the sulfate transporter DTDST. ${ }^{25}$ In addition to the autosomal dominant inherited forms of MED and BHMED, an autosomal recessive spondylo-epi-metaphyseal dysplasia (SEMD; MIM 608728) has been reported in a large consanguineous family of Arabic origin where the mutation is located in the first EGF-like domain of matrilin-3. ${ }^{26} \mathrm{~A}$ second mutation in the first EGF-like domain was found in a genomewide scan to identify candidate genes for hand osteoarthritis (HOA; MIM 607850). ${ }^{27}$ Interestingly, increased levels of matrilin-3 have been detected in ostoarthritic cartilage with a strong correlation between enhanced protein expression and the extent of tissue damage. ${ }^{28}$ Together, these findings indicate an important role of matrilin-3 in the development and homeostasis of cartilage.

In the present study, we have investigated three disease causing mutations located either in the vWA domain or in the first EGF-like domain of matrilin-3. The mutation in the vWA domain causes MED, ${ }^{18}$ whereas the two selected mutations in the EGF-like domains, lying in the immediate vicinity, are linked to $\mathrm{HOA}^{27}$ and SEMD, respectively. ${ }^{26}$ Expression of the mutated constructs in primary chondrocytes gives an insight into the mechanisms involved in the pathogenesis of these diseases at the cellular level and reveals similarities and differences regarding expression and extracellular deposition.

\section{METHODS}

\section{Site directed mutagenesis}

Mutagenesis was performed on a murine full length matrilin3 clone using the QuickChange mutagenesis kit (Stratagene,

Abbreviations: BHMED, bilateral hereditary micro-epiphyseal dysplasia; COMP, cartilage oligomeric matrix protein; ECM, extracellular matrix; EGF, epidermal growth factor; ER, endoplasmatic reticulum; HOA, hand osteoarthritis; HRP, horse radish peroxidase; MED, multiple epiphyseal dysplasia; PDI, protein disulfide isomerase; PSACH, pseudoachondroplasia; SEMD, spondylo-epi-metaphyseal dysplasia; vWA, von Willebrand factor $A$ 
La Jolla, CA). ${ }^{10}$ The mutations were introduced into the mouse sequence according to the patient derived mutations in the human sequence, resulting in the exchanges R116W, T298M, and C299S in mouse corresponding to R121W, T303M, and C304S in human. The following primer pairs were used: 5' -CTTGAACGCTGATGGGAAAATGTGCTCAGCC ATTGATAAGTGTGC-3' and 5' -CACACTTATCAATGGCTGAGC ACATTTTCCCATCAGCGTTC-3' for T298M (new site for $\overline{B l p \mathrm{I})} ;$ 5'-CCACAGACACGTGGGTGGCTGTGGTGAACTATG-3' and 5'-CATAGTTCACCACAGCCACCCACGTGTCTGTGG-3' for R116W; 5'-GAACGCTGATGGGAAAACGTCTTCCGCCATTGAT AAGTGTGC-3' and 5'-GCACACTTATCAATGGCGGAAGACG TTTTCCCATCAGCGTTC-3' for C299S (new site for Eci I). Underlines indicate the mutation and italics an additional silent mutation to identify positive clones by restriction analysis. The mutated constructs were inserted into the episomal expression vector pCEP-Pu-StrepII-tag (C-terminal) in-frame with the sequence of the signal peptide of BM-40 as described earlier. ${ }^{10}$

\section{Culture of primary bovine chondrocytes}

Primary bovine articular chondrocytes were isolated by enzymatic digestion from shoulder joints of 18-24 month old animals as described earlier. ${ }^{29}$ Chondrocytes were cultured in monolayer for 5 days before gene transfer and cultures were analysed 3 days later. The culture medium consisting of DMEM/F12 supplemented with 10\% fetal calf serum was changed twice weekly. Ascorbate $(100 \mu \mathrm{M})$ was added to the culture medium to enhance collagen biosynthesis.

\section{Transfection and expression of wildtype and mutated matrilin-3 constructs}

The gene transfer into primary chondrocytes was optimised in earlier studies. Using the episomal expression vector pCEP$\mathrm{Pu}$ and the non-liposomal transfection reagent FuGENE6 (Roche, Indianapolis, IN), a transfection efficiency of up to $30 \%$ was achieved..$^{30}$ Plasmids encoding for wildtype and mutated matrilin-3 constructs were transfected using FuGENE6 according to the manufacturer's instructions. Secretion of recombinant proteins into the cell culture medium was analysed by SDS-PAGE of medium samples and cell extracts from transfected cells followed by immunoblotting.

\section{SDS-polyacrylamide gel electrophoresis and immunoblotting}

SDS-polyacrylamide gel electrophoresis was performed as described by Laemmli. ${ }^{31}$ For immunoblots the proteins were transferred to nitrocellulose and incubated with an affinity purified rabbit antibody directed against recombinantly expressed mouse matrilin-3 diluted in TBS containing 5\% low fat milk powder. ${ }^{4}$ Bound antibodies were detected by luminescence using horse radish peroxidase (HRP) conjugated swine anti-rabbit IgG (DAKO, Glostrup, Denmark), 3aminophthalhydrazide $(1.25 \mathrm{mM}), \quad p$-coumaric acid (225 $\mathrm{mM})$, and $0.01 \% \mathrm{H}_{2} \mathrm{O}_{2}$.

\section{Immunofluorescence staining of chondrocytes}

Cells were plated and transfected in chamber slides (Nunc, Wiesbaden, Germany) and grown for a further 3 days. After fixation with $2 \%$ paraformaldehyde and permeabilisation using $0.2 \%$ Triton X-100 in PBS and three washes with PBS, the cultures were treated with $10 \%$ normal goat serum in PBS as blocking reagent. The cells were incubated with primary antibodies at a dilution of 1:1000 for $60 \mathrm{~min}$, followed by the detection with a secondary antibody for a further $60 \mathrm{~min}$. Primary antibodies directed against protein disulfide isomerase (PDI) as a marker for the endoplasmatic reticulum (ER) and the $58 \mathrm{~K}$ protein as a marker for the Golgi apparatus were purchased from Biomol (Hamburg, Germany) and Sigma (Munich, Germany), respectively. The affinity purified matrilin-3 antibody was described earlier. ${ }^{4}$ Secondary antirabbit IgG HRP conjugated antibodies were from DAKO and secondary anti-mouse Cy3- and Alexa488-conjugated antibodies were from Molecular Probes (Leiden, Netherlands). The slides were finally mounted in DAKO fluorescent mounting medium and examined under an Axiophot fluorescence microscope (Zeiss, Oberkochen, Germany).

\section{RESULTS AND DISCUSSION}

The chondrodysplasia causing mutations R116W and C299S, and the mutation T298M, linked to HOA, were introduced into the matrilin-3 sequence by site directed mutagenesis and confirmed by sequencing (fig 1). For eukaryotic expression, these matrilin-3 cDNAs were cloned into the expression vector pCEP-Pu. ${ }^{32}$ Then 5 days after isolation, the chondrocytes were transfected transiently. The cell culture supernatant and the cell layer were harvested after 3 days and analysed by SDS-PAGE under non-reducing conditions. In non-transfected chondrocytes we could not detect any endogenous matrilin-3, although it has been demonstrated previously that the antibody reacts with both mouse and bovine matrilin-3 (see supplementary fig 1 available at http:// jmedgenet.com/supplemental). ${ }^{4}$ This suggests that only small amounts of endogenous matrilin-3, if any, are expressed in primary bovine chondrocytes isolated from adult animals and that the formation of a significant amount of heterooligomers consisting of endogenous and transfected matrilin-3 is unlikely in our model. In autosomal dominant forms of chondrodysplasia, a certain amount of matrilin-3 tetramers consisting of four mutated subunits will exist and contribute significantly to the phenotype, even though most tetramers are likely to be mixed. In the case of the C299S mutation, which represents an autosomal recessive disease, the absence of wildtype matrilin-3 and thus of heterooligomers consisting of wildtype and mutated subunits, reflects the pathophysiological situation.

In cells transfected with the wildtype construct, matrilin-3 appears as an intact tetramer with an apparent molecular mass of approximately $200 \mathrm{kDa}$ and, in addition, as a trimer where most of one subunit has been cleaved off by proteolytic processing (fig 2; see also supplementary fig 2 available at http://jmedgenet.com/supplemental). ${ }^{13}$ This processing occurs also in cells transfected with the mutant T298M. In cell extracts, we mainly found the tetrameric protein which is partially cleaved to trimers when it appears in the supernatant. Expression levels and localisation of the T298M mutant were comparable to wildtype, whereas the other two mutants were scarcely secreted and mainly detected in the cell extracts. The comparatively weak signal for the mutant proteins R116W and C299S in cell extracts does not allow any conclusion with regard to proteolytic processing.

The low expression level of R116W in chondrocytes might be due to a rapid intracellular degradation as shown for a glycine to glutamic acid mutation in the collagen VI $\alpha 3$ chain (leading to Bethlem myopathy), which is also located in the $\beta 2$ strand of the vWA domain N2. ${ }^{33}$ This mutation interferes with protein folding and due to intracellular degradation patient derived fibroblasts secrete low levels of collagen VI.

The amino acid exchange C299S affects a highly conserved cysteine within the first EGF repeat and the mutant protein is also retained intracellularly. The uneven number of cysteines resulting from this exchange might lead to disulfide shuffling. This may have severe functional and structural consequences as demonstrated recently for mutations in the EGF domains of fibrillin-l and factor IX. Cysteine mutations in recombinantly expressed fibrillin-1 enhanced 


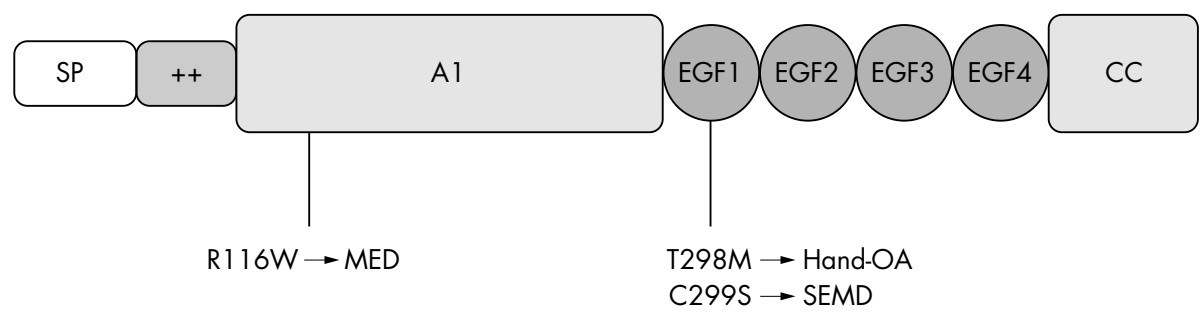

Figure 1 Domain structure of matrilin-3 and localisation of the mutations introduced in the present study. The mutations were introduced into the mouse sequence according to the patient derived mutations in the human sequence with the exchanges R116W, T298M, and C299S in mouse corresponding to R121W, T303M, and C304S in human. ${ }^{18}{ }^{26}{ }^{27}$ The position of the mutation and the resulting disease phenotype is indicated. ++, positively charged stretch; Al, von Willebrand factor A domain; CC, coiled coil; EGF, epidermal growth factor-like domain; MED, multiple epiphyseal dysplasia; OA, osteoarthritis; SEMD, spondylo-epi-metaphyseal dysplasia; SP, signal peptide.

the susceptibility for proteolytic degradation and it may be that a lack of extracellular fibrillin plays an important role in the development of fibrillopathies. ${ }^{34}$

In human factor IX, patient mutations dramatically impair intracellular processing and secretion, which explains the highly reduced factor IX plasma concentrations. ${ }^{35}$ So far, five mutations within four of the six $\beta$ strand regions of the single vWA domain of matrilin-3 have been described, with the arginine to tryptophan exchange being the most frequent. ${ }^{1936}$ Molecular modelling and comparison with the I domain of integrin $\alpha$ l has led to the assumption that the amino acid exchanges in the vWA domain either have an influence on the metal ion dependent adhesion site or affect the folding of the domain by introducing bulky side chains like, for example, tryptophan. ${ }^{18}{ }^{19}$ Arginine 116 is conserved in all matrilins as well as in the Al domain of the von Willebrand factor. The crystal structure of the vWAl domain has been solved showing that this arginine 552 is involved in the formation of salt bridges. ${ }^{37}$ Interestingly, a patient derived arginine to cysteine mutation at exactly this position leads to an abnormal folding of the von Willebrand factor with a loss of function. ${ }^{38}$ Other point mutations in the vWA domains of the von Willebrand factor have been shown to induce conformational changes affecting its multimeric structure and resulting in abnormal binding to collagen. ${ }^{39} 40$

For a more detailed analysis of the intra- and extracellular localisation of the recombinant proteins, immunofluorescence staining was performed on transfected chondrocytes. A costaining with antibodies directed against PDI and the 58K protein as marker proteins for the ER and the Golgi apparatus allowed the assignment of the matrilin-3 staining to a specific intracellular compartment. In chondrocytes, the wildtype protein and the T298M mutant co-localise with the perinuclear Golgi apparatus, demonstrating that the intracellular trafficking of these proteins from ER to Golgi apparatus is not affected by the mutation (fig 3A and B). In contrast, the mutant proteins R116W and C299S are mainly detected in the ER which is typically spread throughout the whole cell (fig $3 \mathrm{C}$ and D). There is no overlapping staining for matrilin-3 and the Golgi compartment, suggesting that the protein remains trapped in the ER. Defects in intracellular trafficking leading to retention and accumulation of cartilage proteins in the ER have been described for mutated variants of the ( $\alpha 3)$ chain of collagen type IX and cartilage oligomeric matrix protein both in patient cartilage and in cell culture models. ${ }^{41-44}$ Mutations in both proteins lead to very similar disease phenotypes suggesting that retention and accumulation of cartilage proteins in the ER might be a general mechanism involved in the pathogenesis of chondrodysplasias. At least for mutations in COMP, it has been demonstrated that both intra- and extracellular pathways are involved in the pathogenesis of the disease. While some COMP mutants accumulate in the ER and cause a decrease in cell viability,

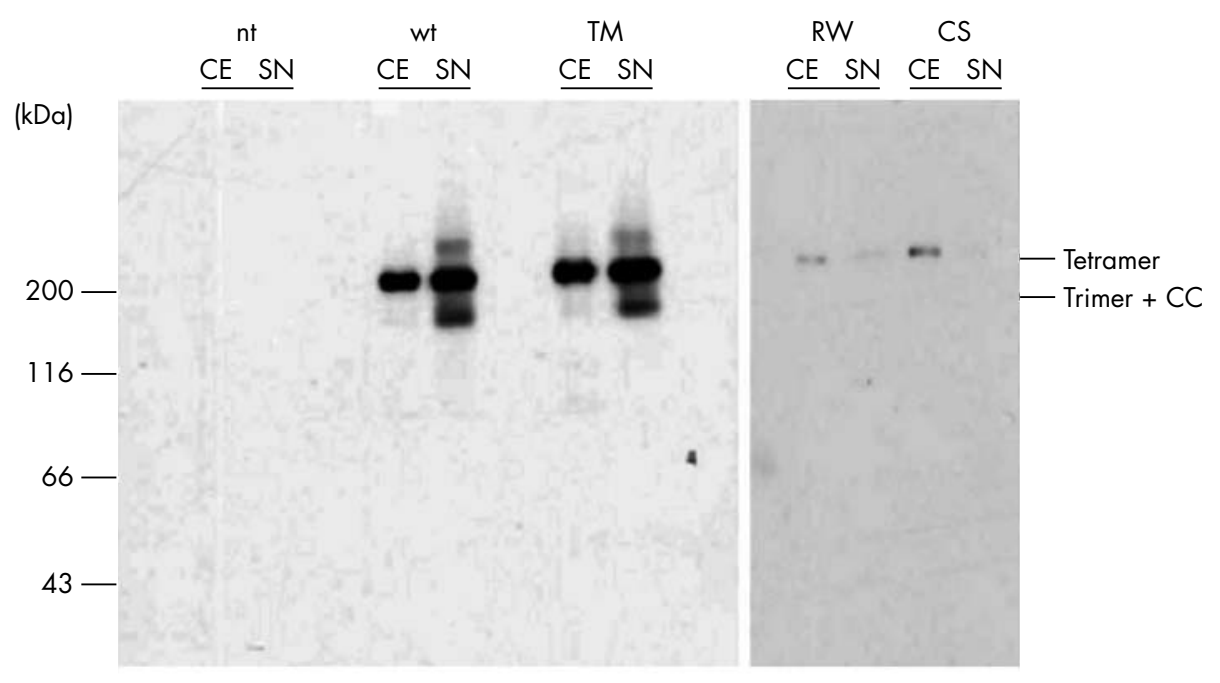

Figure 2 Immunoblot analysis of transfected primary chondrocytes. Cell extracts (CE) and cell culture supernatants (SN) were harvested 3 days after transfection and submitted to SDS-PAGE under non-reducing conditions. Similar amounts of total protein were loaded for each construct. Even after prolonged exposure times (right panel), the constructs R1 16W and C299S were only weakly detected. Mobilities of the protein standards are indicated in kDa. CC, coiled coil. 

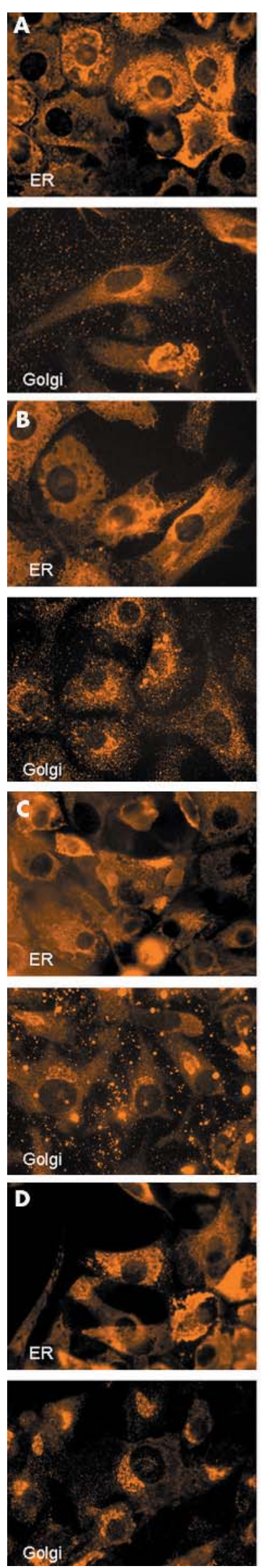
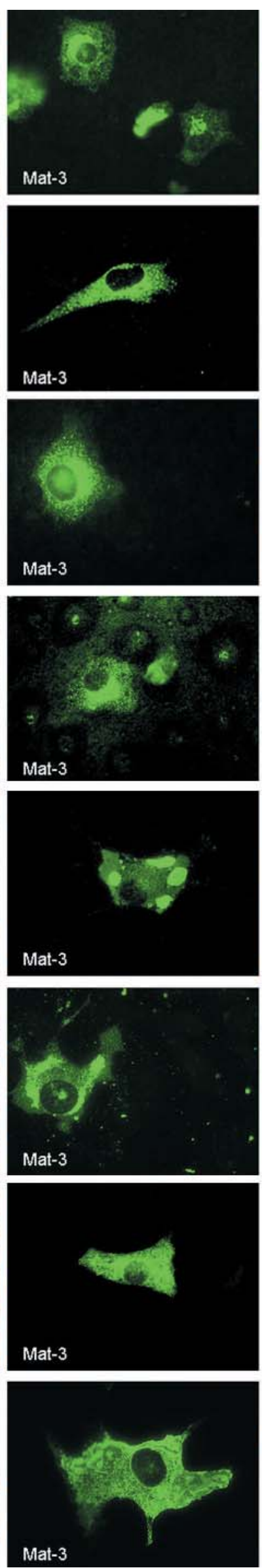
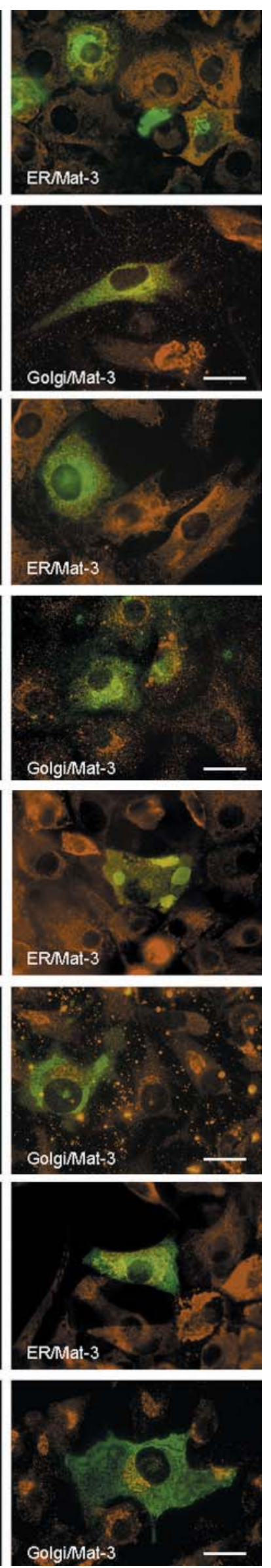

Figure 3 Immunofluorescence staining of primary chondrocytes 3 days after transfection. Cells were transfected with wildtype (A), T298M (B), R116W (C), and C299S matrilin-3 (D) and cultured in the absence of ascorbate to prevent formation of extracellular networks which could obstruct the view of intracellular structures. ER and Golgi apparatus are stained in red using $\mathrm{C}_{\mathrm{C}} 3$ conjugated secondary antibodies directed against PDI (top left) and 58K protein (bottom leff), respectively. Matrilin-3 was visualised in green using an Alexa488 labelled secondary antibody (middle). Images were merged to visualise a potential colocalisation between matrilin-3 proteins with the ER or the Golgi compartment (right). The bar represents $20 \mu \mathrm{m}$. 
others appear to disturb the assembly of the pericellular matrix. ${ }^{44}$ Since the complete absence of either matrilin-3 or COMP does not lead to any obvious phenotypes in mouse, it has been speculated that the mutated protein might exert a dominant negative effect on chondrocyte function and the ECM assembly. ${ }^{17} 45$

We therefore analysed matrilin-3 incorporation into the ECM of cultured chondrocytes. In cells transfected with the wildtype and the T298M construct, we could detect a matrilin-3 positive filamentous network surrounding and connecting cells (fig 4). This is in good agreement with previous results for chondrosarcoma cells. The formation of these structures was dependent on the presence of ascorbate in the culture medium, suggesting that matrilin-3 is mainly incorporated into a collagenous network. ${ }^{4}$ Linkage of the T298M matrilin-3 mutation with the development of HOA is controversial. This mutation and another substitution in an $\alpha$-helical region of the vWA domain have also been classified as non-synonymous polymorphisms. ${ }^{19}$ Our results show on the cellular level that T298M is secreted normally and incorporated into the ECM in a manner similar to the wildtype matrilin-3. Although it can not be completely excluded that the T298M mutation has a minor effect on the structure and function of matrilin-3, our findings corroborate the hypothesis that, at least, additional factors are needed for the development of HOA.

In cells transfected with the mutants R116W and C299S, the filamentous structures were completely absent (fig 4). This demonstrates that mutated matrilin-3, if secreted at all, neither forms fibrillar structures itself nor interacts with existing collagen networks. In addition to the lack of extracellular staining, we observed inclusion bodies in chondrocytes transfected with Rl16W (figs 3C and 4). These structures closely resemble the inclusions described in chondrocytes of MED and pseudoachondroplasia (PSACH) patients with mutations in collagen type IX and COMP, and it might well be that these structures interfere with the secretion of other matrix components, for example, aggrecan and collagen type IX, perhaps through interactions with matrilin-3. ${ }^{41-43}$ The massive accumulation of protein could not only compromise protein secretion but could also affect cell viability through induction of apoptosis as demonstrated in PSACH chondrocytes. ${ }^{46}$ Since the highest expression levels of matrilin-3 in the growth plate have been detected in the proliferation zone, ${ }^{47}$ it is attractive to speculate that cell death induced by mutations in matrilin-3 could contribute to reduced growth of the long bones.
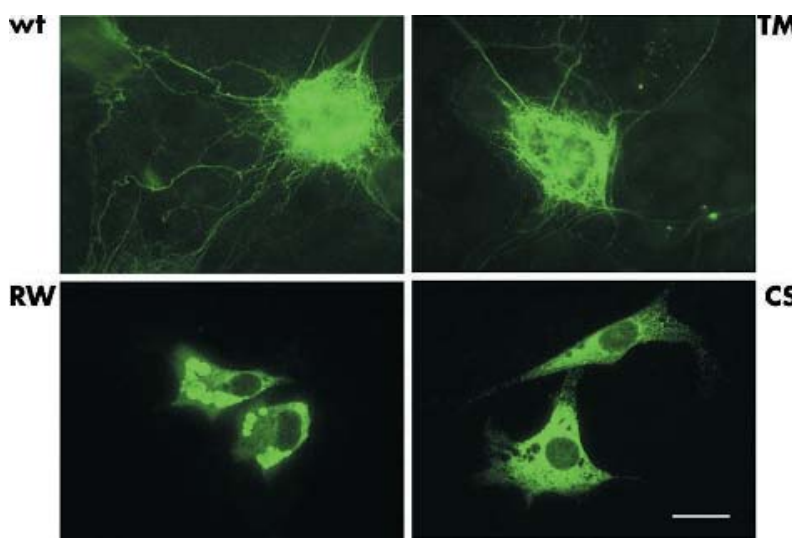

Figure 4 Immunofluorescence staining of primary chondrocytes 3 days after transfection. Cells were cultured in the presence of ascorbate to allow formation of extracellular filamentous structures. Matrilin-3 was visualised in green using a secondary Alexa488 labelled secondary antibody. The bar represents $20 \mu \mathrm{m}$.
In summary, our results show that mutations in matrilin-3 causing chondrodysplasias (R116W and C299S) interfere with intracellular protein trafficking and formation of filamentous extracellular structures. Since the matrilin-3 deficient mouse does not show any obvious skeletal abnormalities, we conclude that these mutations exert their action mainly through a dominant negative mechanism. In contrast, matrilin-3 carrying a mutation linked to HOA (T298M) is expressed, processed, secreted, and incorporated in an extracellular network in a way indistinguishable from the wildtype protein, suggesting only subtle, if any, effects on the structure and function of the protein.

\section{ELECTRONIC-DATABASE INFORMATION}

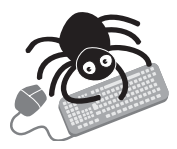

Analysis of the species specificity of the matrilin-3 antibody and immunoblot analysis of transfected primary chondrocytes are shown in supplementary figures available at http://jmedgenet.com/ supplemental.

\section{Authors' affiliations}

C Otten, R Wagener, M Paulsson, F Zaucke, Center for Biochemistry, Medical Faculty, University of Cologne, D-50931 Cologne, Germany M Paulsson, Center for Molecular Medicine, Medical Faculty, University of Cologne, D-50931 Cologne, Germany

$\mathrm{CO}$ and $\mathrm{FZ}$ were supported by the Köln Fortune programme/Faculty of Medicine, University of Cologne and RW and MP by the Deutsche Forschungsgemeinschaft (WA 1338/2-2 and 1338/2-3).

Competing interests: none declared

Correspondence to: Frank Zaucke, Center for Biochemistry, Medical Faculty, University of Cologne, Joseph-Stelzmann-Str. 52, D-50931 Cologne, Germany; frank.zaucke@uni-koeln.de

Received 21 November 2004

Revised version received 25 January 2005

Accepted for publication 3 February 2005

\section{REFERENCES}

1 Deák F, Wagener R, Kiss I, Paulsson M. The matrilins: a novel family of oligomeric extracellular matrix proteins. Matrix Biol 1999;18:55-64.

2 Wagener R, Kobbe B, Paulsson M. Primary structure of matrilin-3, a new member of a family of extracellular matrix proteins related to cartilage matrix protein (matrilin-1) and von Willebrand factor. FEBS Lett 1997;413:129-34.

3 Wu JJ, Eyre D. Matrilin-3 forms disulfide-linked oligomers with matrilin-1 in bovine epiphyseal cartilage. J Biol Chem 1998;273:17433-8.

4 Klatt AR, Nitsche DP, Kobbe B, Mörgelin M, Paulsson M, Wagener R. Molecular structure and tissue distribution of matrilin-3, a filament-forming extracellular matrix protein expressed during skeletal development. J Biol Chem 2000;275:3999-4006.

5 Kleemann-Fischer D, Kleemann GR, Engel D, Yates JR 3rd, Wu JJ, Eyre DR. olecular properties of matrilin-3 isolated from human growth cartilage. Arch Biochem Biophys 2001;387:209-15.

6 Hauser N, Paulsson M, Heinegård D, Mörgelin M. Interaction of cartilage matrix protein with aggrecan. Increased covalent cross-linking with tissue maturation. J Biol Chem 1996;271:32247-52.

7 Winterbottom N, Tondravi MM, Harrington TL, Klier FG, Vertel BM, Goetinck PF. Cartilage matrix protein is a component of the collagen fibril of cartilage. Dev Dyn 1992;193:266-76.

8 Piecha D, Wiberg C, Morgelin M, Reinhardt DP, Deák F, Maurer P, Paulsson M. Matrilin-2 interacts with itself and with other extracellular matrix proteins. Biochem J 2002;367:715-21.

9 Wiberg $C$, Klatt AR, Wagener R, Paulsson M, Bateman JF, Heinegård D, Mörgelin M. Complexes of matrilin-1 and biglycan or decorin connect collagen VI microfibrils to both collagen II and aggrecan. J Biol Chem 2003;278:37698-704.

10 Mann HH, Özbek S, Engel J, Paulsson M, Wagener R. Interactions between the cartilage oligomeric matrix protein and matrilins. Implications for matrix assembly and the pathogenesis of chondrodysplasias. J Biol Chem assembly and the path;279:25294-8.

11 Chen Q, Johnson DM, Haudenschild DR, Tondravi MM, Goetinck PF. Cartilage matrix protein forms a type II collagen-independent filamentous network: analysis in primary cell cultures with a retrovirus expression system. Mol Biol Cell 1995;6:1743-53.

12 Piecha D, Muratoglu S, Mörgelin M, Hauser N, Studer D, Kiss I, Paulsson M, Deák F. Matrilin-2, a large, oligomeric matrix protein, is expressed by a great 
variety of cells and forms fibrillar networks. J Biol Chem 1999;274:13353-61.

13 Klatt AR, Nitsche DP, Kobbe B, Macht M, Paulsson M, Wagener R. Molecular structure, processing, and tissue distribution of matrilin-4. J Biol Chem 2001; 276:17267-75.

14 Huang X, Birk DE, Goetinck PF. Mice lacking matrilin-1 (cartilage matrix protein) have alterations in type II collagen fibrillogenesis and fibril organization. Dev Dyn 1999;216:434-41

15 Aszódi A, Bateman JF, Hirsch E, Baranyi M, Hunziker E, Hauser N, Bösze Z, Fässler R. Normal skeletal development of mice lacking matrilin 1: redundant function of matrilins in cartilage? Mol Cell Biol 1999;19:7841-5

16 Mátés L, Nicolae C, Mörgelin M, Deák F, Kiss I, Aszódi A. Mice lacking the extracellular matrix adaptor protein matrilin-2 develop without obvious abnormalities. Matrix Biol 2004;23:195-204.

17 Ko Y, Kobbe B, Nicolae C, Miosge N, Paulsson M, Wagener R, Aszódi A. Matrilin-3 is dispensable for mouse skeletal growth and development. Mol Cell Biol 2004; 24:1691-9.

18 Chapman KL, Mortier GR, Chapman K, Loughlin J, Grant ME, Briggs MD. Mutations in the region encoding the von Willebrand factor A domain of matrilin-3 are associated with multiple epiphyseal dysplasia. Nat Genet $2001 ; 28: 393-6$.

19 Jackson GC, Barker FS, Jakkula E, Czarny-Ratajczak M, Makitie O, Cole WG, Wright MJ, Smithson SF, Suri M, Rogala P, Mortier GR, Baldock C, Wallace A Elles R, Ala-Kokko L, Briggs MD. Missense mutations in the beta strands of the single A-domain of matrilin-3 result in multiple epiphyseal dysplasia. J Med Genet 2004;41:52-9

20 Mostert AK, Dijkstra PF, Jansen BR, van Horn JR, de Graaf B, Heutink P, Lindhout $D$. Familial multiple epiphyseal dysplasia due to a matrilin-3 mutation: further delineation of the phenotype including 40 years follow-up. Am J Med Genet 2003;120A:490-7.

21 Muragaki Y, Mariman EC, van Beersum SE, Perala M, van Mourik JB, Warman ML, Hamel BC, Olsen BR. A mutation in COL9A2 causes multiple epiphyseal dysplasia (EDM2). Ann N Y Acad Sci 1996;785:303-6.

22 Paassilta P, Lohiniva J, Annunen S, Bonaventure J, Merrer ML, Pai L, AlaKokko L. COL9A3: a third locus for multiple epiphyseal dysplasia. Am J Hum Genet 1999;64:1036-44.

23 Czarny-Ratajczak M, Lohiniva J, Rogala P, Kozlowski K, Perälä M, Carter L, Spector TD, Kolodziej L, Seppänen U, Glazar R, Krolewski J, LatosBielenska A, Ala-Kokko L. A mutation in COL9A1 causes multiple epiphyseal dysplasia: further evidence for locus heterogeneity. Am J Hum Genet 2001;69:969-80.

24 Briggs MD, Hoffman SM, King LM, Olsen AS, Mohrenweiser H, Leroy JG, Mortier GR, Rimoin DL, Lachman RS, Gaines ES, Cekleniak JA, Knowlton RG, Cohn DH. Pseudoachondroplasia and multiple epiphyseal dysplasia due to mutations in the cartilage oligomeric matrix protein gene. Nat Genet 1995; 10:330-6.

25 Rossi A, Superti-Furga A. Mutations in the diastrophic dysplasia sulfate transporter (DTDST) gene (SLC26A2): 22 novel mutations, mutation review, associated skeletal phenotypes, and diagnostic relevance. Hum Mutat 2001;17:159-71.

26 Borochowitz ZU, Scheffer D, Adir V, Dagoneau N, Munnich A, CormierDaire V. Spondylo-epi-metaphyseal dysplasia (SEMD) matrilin 3 type: homozygote matrilin 3 mutation in a novel form of SEMD. J Med Genet 2004;41:366-72.

27 Stefansson SE, Jonsson $\mathrm{H}$, Ingvarsson T, Manolescu I, Jonsson $\mathrm{HH}$, Olafsdottir G, Palsdottir E, Stefansdottir G, Sveinbjornsdottir G, Frigge ML, Kong A, Gulcher JR, Stefansson K. Genomewide scan for hand osteoarthritis: a novel mutation in matrilin-3. Am J Hum Genet 2003;72:1448-59.

28 Pullig O, Weseloh G, Klatt AR, Wagener R, Swoboda B. Matrilin-3 in human articular cartilage: increased expression in osteoarthritis. Osteoarthritis Cartilage 2002; 10:253-63.

29 Zaucke F, Dinser R, Maurer P, Paulsson M. Cartilage oligomeric matrix protein (COMP) and collagen IX are sensitive markers for the differentiation state of articular primary chondrocytes. Biochem J 2001;358:17-24.

30 Dinser R, Kreppel F, Zaucke F, Blank C, Paulsson M, Kochanek S, Maurer P. Long-term transgene expression in primary articular chondrocytes following nonviral and adenoviral gene transfer. Histochem Cell Biol 2001;1 16:69-77.
31 Laemmli UK. Cleavage of structural proteins during the assembly of the head of bacteriophage T4. Nature 1970;227:680-5.

32 Kohfeldt E, Maurer P, Vannahme C, Timpl R. Properties of the extracellular calcium binding module of the proteoglycan testican. FEBS Lett 1997;414:557-61.

33 Sasaki T, Hohenester E, Zhang RZ, Gotta S, Speer MC, Tandan R, Timpl R, Chu ML. A Bethlem myopathy Gly to Glu mutation in the von Willebrand factor $\mathrm{A}$ domain $\mathrm{N} 2$ of the collagen alpha3(VI) chain interferes with protein folding. FASEB J 2000;14:761-8

34 Vollbrandt T, Tiedemann K, El-Hallous E, Lin G, Brinckmann J, John H, Batge B, Notbohm H, Reinhardt DP. Consequences of cysteine mutations in calcium-binding epidermal growth factor modules of fibrillin-1. J Biol Chem 2004; 279:32924-31

35 Enjolras N, Plantier JL, Rodriguez MH, Rea M, Attali O, Vinciguerra C, Negrier C. Two novel mutations in EGF-like domains of human factor IX dramatically impair intracellular processing and secretion. J Thromb Haemost 2004; 2: 1143-54.

36 Mäkitie O, Mortier GR, Czarny-Ratajczak M, Wright MJ, Suri M, Rogala P, Freund M, Jackson GC, Jakkula E, Ala-Kokko L, Briggs MD, Cole WG. Clinical and radiographic findings in multiple epiphyseal dysplasia caused by MATN3 mutations: description of 12 patients. Am J Med Genet 2004; 125A:278-84.

37 Emsley J, Cruz M, Handin R, Liddington R. Crystal structure of the von Willebrand Factor $\mathrm{Al}$ domain and implications for the binding of platelet glycoprotein Ib. J Biol Chem 1998;273:10396-401.

38 Ribba AS, Hilbert L, Lavergne JM, Fressinaud E, Boyer-Neumann C, Ternisien C, Juhan-Vague I, Goudemand J, Girma J, Mazurier C, Meyer D. The arginine-552-cysteine (R1315C) mutation within the Al loop of von Willebrand factor induces an abnormal folding with a loss of function resulting in type 2A-like phenotype of von Willebrand disease: study of 10 patients and mutated recombinant von Willebrand factor. Blood 2001;97:952-9

39 Ribba AS, Loisel I, Lavergne JM, Juhan-Vague I, Obert B, Cherel G, Meyer D, Girma J. Ser968Thr mutation within the A3 domain of von Willebrand factor (VWF) in two related patients leads to a defective binding of WWF to collagen. Thromb Haemost 2001;86:848-54.

40 Stepanian A, Ribba AS, Lavergne JM, Fressinaud E, Juhan-Vague I, Mazurier C, Girma JP, Meyer D. A new mutation, S1285F, within the A1 loop of von Willebrand factor induces a conformational change in A1 loop with abnormal binding to platelet GPlb and botrocetin causing type $2 \mathrm{M}$ von Willebrand disease. Br J Haematol 2003;120:643-51.

41 Vranka J, Mokashi A, Keene DR, Tufa S, Corson G, Sussman M, Horton WA, Maddox K, Sakai L, Bächinger HP. Selective intracellular retention of extracellular matrix proteins and chaperones associated with pseudoachondroplasia. Matrix Biol 2001;20:439-50.

42 Maddox BK, Keene DR, Sakai LY, Charbonneau NL, Morris NP, Ridgeway CC, Boswell BA, Sussman MD, Horton WA, Bächinger HP, Hecht JT. The fate of cartilage oligomeric matrix protein is determined by the cell type in the case of a novel mutation in pseudoachondroplasia. J Biol Chem 1997;272:30993-7.

43 Bönnemann CG, Cox GF, Shapiro F, Wu JJ, Feener CA, Thompson TG, Anthony DC, Eyre DR, Darras BT, Kunkel LM. A mutation in the alpha 3 chain of type IX collagen causes autosomal dominant multiple epiphyseal dysplasia with mild myopathy. Proc Natl Acad Sci U S A 2000;97:1212-7.

44 Dinser R, Zaucke F, Kreppel F, Hultenby K, Kochanek S, Paulsson M, Maurer P. Pseudoachondroplasia is caused through both intra- and extracellular pathogenic pathways. J Clin Invest 2002;1 10:505-13.

45 Svensson L, Aszódi A, Heinegård D, Hunziker EB, Reinholt FP, Fässler R, Oldberg $\AA$. Cartilage oligomeric matrix protein-deficient mice have normal skeletal development. Mol Cell Biol 2002;22:4366-71.

46 Duke J, Montufar-Solis D, Underwood S, Lalani Z, Hecht JT. Apoptosis staining in cultured pseudoachondroplasia chondrocytes. Apoptosis 2003;8:191-7.

47 Zhang $Y$, Chen $Q$. Changes of matrilin forms during endochondral ossification. Molecular basis of oligomeric assembly. J Biol Chem 2000;275:32628-34. 